\title{
Inserción de un paciente estandarizado como estrategia didáctica en Odontología
}

\author{
Insertion of a standardized patient as a didactic strategy in dentistry \\ Mario Zúñiga-Mogollones,* Giorgina Ferri-Sánchez,** \\ Carolina Oviedo-Sarmiento, * Carolina Baltera-Zuloaga*
}
Palabras clave: Paciente estandarizado, simulación, odontología, autoeficacia, retención.
Key words: Patient standardized, simulation, odontology, self-efficacy, student retention.

* Cirujano dentista. Magister en Educación en Ciencias de la Salud. ** Cirujana dentista. Magister en Pedagogía Universitaria.

Facultad de Odontología, Universidad San Sebastián.

\section{RESUMEN}

Las asignaturas clínicas en Odontología presentan la más alta tasa de reprobación de la carrera, lo que repercute en la titulación efectiva y la retención estudiantil. Los estudiantes sufren altos niveles de estrés, depresión y ansiedad al ser enfrentados por primera vez a pacientes reales, sin haber tenido un entrenamiento relacional previo. La estrategia didáctica de paciente estandarizado de alta fidelidad se inserta por primera vez en la carrera para fortalecer las competencias necesarias para el tratamiento de individuos en sus tres aspectos fundamentales, las cuales incluyen el procesamiento de una ficha clínica endodóntica, la formulación del diagnóstico de la patología pulpo-periapical con base en la integración de la información clínica y los exámenes complementarios, y el trato empático de los pacientes. Esta intervención estuvo dirigida a 120 estudiantes de tercer año de la asignatura Semiología del ciclo preclínico. El trabajo cumplió las siguientes etapas: el briefing, el escenario, el debriefing y la evaluación del impacto de la estrategia a través de la percepción del escenario por parte del estudiante y la observación directa del desempeño por parte del docente experto. La encuesta realizada a los estudiantes tras la experiencia reportó una alta valoración en el aspecto del desarrollo de habilidades de comunicación (98\%) y la preparación para el desempeño con pacientes reales (95\%), mientras que la pauta de observación docente demostró resultados positivos como la capacidad diagnóstica adecuada (90\%), sistematización de la información de la ficha clínica $(70 \%)$ y un bajo rendimiento en empatía con el paciente (40\%).

\section{ABSTRACT}

Clinical courses in Dentistry present the highest failure rate of the career, having an impact on the graduation rates and student retention. Students suffer high levels of stress, depression and anxiety when confronted for the first time with real patients, without having had a prior relational training. The standardized, high-fidelity didactic patient strategy was inserted for the first time in the curriculum to strengthen the competencies necessary for the treatment of patients with painful pulpoperiapical pathologies, working on the three fundamental aspects that define a competence. This intervention was aimed at 120 third-year students of the Semiology course of the preclinical cycle. The work fulfilled the following stages: the briefing, the scenario, the debriefing and the evaluation of the perception of the scenario by the student and the direct observation of the performance by the expert teacher. The survey made to the students after the experience reported a high valuation in the aspect like the development of communication skills (98\%) and preparation for performance with real patients (95\%), while the teacher observation pattern showed positive results as the adequate diagnostic capacity $(90 \%)$, systematization of the information of the clinical record (70\%) and a low performance in empathy with the patient $(40 \%)$.

\section{INTRODUCCIÓN}

T as asignaturas clínicas de la carrera en $ـ$ nuestra universidad presentan un alta tasa de reprobación, cercana al $45 \%$ promedio de todas las sedes donde se imparte (Santiago, Concepción, Puerto Montt y Valdivia), lo que repercute en los indicadores de calidad del proceso educativo, como tiempo de titulación efectiva y retención estudiantil. En la investigación desarrollada por Jaramillo se evidencia que los estudiantes de cuarto año sufren altos niveles de estrés, depresión y ansiedad al enfrentarse por primera vez como tratantes de pacientes reales, sin un acercamiento o entrenamiento previo, especialmente en aspectos relacionales. ${ }^{1}$ Esta realidad es muy similar a la que han reportado nuestros estudiantes del cuarto año de la carrera.

La simulación clínica es una estrategia didáctica y evaluativa que cada vez cobra 
más fuerza en el currículo de las carreras de la salud debido a su utilidad en el desarrollo de competencias profesionales. ${ }^{2}$ La simulación crea un ambiente ideal para la educación, ya que las actividades pueden diseñarse para que sean predecibles, consistentes, estandarizadas, seguras y reproducibles. ${ }^{3}$

En Odontología, el uso de simulación es parte de las metodologías tradicionalmente utilizadas, en especial en simuladores de baja fidelidad, los cuales sólo desarrollan habilidades psicomotoras antes de la atención a individuos reales. ${ }^{4}$ Existen pocas intervenciones documentadas en Odontología donde el uso de pacientes estandarizados forme parte de instancias formativas en asignaturas preclínicas. Autores como Johnson y sus colaboradores reportan la incorporación de pacientes estandarizados durante el segundo año de la formación de estudiantes de la asignatura Laboratorio de Salud Oral para apoyar el desarrollo de algunas actividades prácticas como la toma de impresiones, el registro de relación céntrica, dimensión vertical y evaluación de la sonrisa, que no podían ser realizadas durante la didáctica de los laboratorios dentales. ${ }^{5}$ Así también, McKenzie y su grupo estudiaron el uso de los pacientes estandarizados en el desarrollo de habilidades comunicacionales en estudiantes previamente a la atención de los consultantes; en este caso, los instructores reportaron que el grupo de estudiantes sometidos a esta experiencia fue capaz de ofrecer más opciones de tratamiento a los pacientes y responder las preguntas sobre estas, pero que se requieren trabajos a largo plazo para evaluar de mejor manera el impacto de esta estrategia. ${ }^{6}$ Una de las grandes ventajas que la literatura reporta sobre esta metodología es la posibilidad de obtener una retroalimentación directa del paciente estandarizado, que aporta en generar mejoras progresivas de las habilidades comunicacionales en estas experiencias, según lo reportado por Wagner y sus colegas en una experiencia realizada en la Universidad Arizona, en la Escuela de Medicina Dental. ${ }^{7}$

En la carrera de Odontología de la Universidad San Sebastián, esta intervención con simulación clínica resulta ser el primer acercamiento que expone de manera temprana a los estudiantes del ciclo preclínico a una fidelidad media a alta, con la intención de evidenciar la integración de recursos cognitivos, procedimentales y actitudinales en una misma instancia formativa.

\section{MATERIAL Y MÉTODOS}

Se diseñó un escenario de simulación clínica con paciente estandarizado, inserto como estrategia metodológica de la unidad "Diagnóstico pulpar y periapical" de la asignatura Semiología en el tercer año de la carrera de Odontología en la sede Santiago, en el cual finaliza el ciclo de formación preclínica, previo a la atención clínica.

La intervención fue dirigida a 120 estudiantes inscritos en la asignatura; fue realizada en las horas de práctica (o laboratorio) con una proporción docente-estudiante de 1:8, divididos en 15 subsecciones. La muestra de estudio correspondió a la totalidad de los estudiantes de la asignatura de Semiología.

El resultado de aprendizaje esperado, seleccionado a partir del programa de semiología, fue: "diagnostica enfermedades pulpo-periapicales en un paciente adulto con patología dolorosa en un ambiente simulado". Este se eligió a partir de la posibilidad de generar un escenario con paciente estandarizado, debido a la alta demanda de urgencias odontológicas asociadas al dolor de origen dentario y a la posibilidad de evaluar en un escenario desempeños cognitivos, procedimentales y actitudinales, esto sumado a informaciones referidas por los tutores clínicos en reuniones de comunidades académicas que indicaban que los estudiantes presentaban dificultades diagnósticas y de empatía con estos pacientes en el área clínica de la carrera.

Los desempeños por demostrar durante el desarrollo del escenario fueron: manejo de la ficha clínica, diagnóstico y empatía con el paciente, evaluados a través de una pauta conocida por los estudiantes y por el docente.

Previamente a la actividad, los estudiantes asistieron a las clases teóricas, en donde se trabajó a través de clases magistrales y resolución de casos clínicos, y se les entregaron los contenidos teóricos declarados en el programa de la asignatura.

El trabajo durante la sesión de simulación clínica se desarrolló en las siguientes etapas:

1) Briefing (sesión informativa), donde los estudiantes, guiados por el docente, revisaron 
los contenidos teóricos, reforzados a través de una lectura complementaria (indicada en la guía del estudiante) que fue compartida antes de la realización de la experiencia. Posteriormente, se procedió a explicar la finalidad de la intervención, a través de socializar con los estudiantes el resultado de aprendizaje esperado, así como los desempeños a demostrar.

2) Se firmó un compromiso de confidencialidad con respecto al desarrollo del escenario, para cuidar el traspaso de información y vivencias entre los grupos aún no expuestos a la actividad.

3) El escenario simulado se realizó en el cubículo de atención dental del Hospital de Simulación de la Universidad San Sebastián con la participación de ocho estudiantes distribuidos en 15 subsecciones de práctica, donde se distribuyeron los siguientes roles: dos estudiantes voluntarios sometidos al escenario, una actriz como paciente estandarizada y un confederado.

4) Los restantes seis estudiantes y el docente se encontraban en una sala realizando una observación indirecta del desarrollo de la actividad, completando la pauta de cotejo construida por los docentes de Semiología a partir de los desempeños esperados de evaluación. Esta pauta se encontraba adjunta a la guía del estudiante, para hacerlos participar en el debriefing (sesión informativa). La duración del escenario fue de 12 minutos aproximadamente.

5) Debriefing. Se realizó una evaluación formativa y participativa a nivel grupal sobre los aspectos positivos y a mejorar del desempeño demostrado por los estudiantes participantes. Esta instancia sirvió de reflexión grupal en torno a la experiencia de enfrentarse a un paciente real y contó con la participación de la actriz, quien comentó cómo se sintió ella con el manejo de la situación por parte de los estudiantes, lo que permitió valorar la importancia de la empatía y las relaciones humanas.

6) Aplicación del instrumento de evaluación de la intervención, el cual fue una encuesta de percepción basada en una escala de Likert, estandarizada y provista por el centro de simulación USS junto a un anexo de preguntas abiertas con respecto a su percepción y el impacto de esta estrategia en el desarrollo de sus competencias; este fue valorado cualitati- vamente a través de un análisis de discurso.

\section{RESULTADOS}

La presentación de los resultados se realizó a través de tres componentes:

a) Percepción, componente cuantitativo, que se desprende de la encuesta de satisfacción estandarizada del centro de simulación USS (Tabla 1):

Estos resultados demuestran que la mayor valoración la obtuvo el desarrollo de habilidades de comunicación (98\%), la preparación para el desempeño con pacientes reales (95\%), la confianza en sus capacidades comunicativas (93\%) y el deseo de contar con más actividades de este tipo (93\%). Los ítems menos valorados fueron la obligatoriedad de incorporación al currículum (87\%) y la comodidad con la estrategia (79\%). Cabe destacar que casi todas las respuestas a los ítems se encontraban distribuidas entre los niveles "de acuerdo" y "completamente de acuerdo"; en la suma de estas dos alternativas se obtuvo un $98 \%$ de promedio, en contraparte, las opciones "no está seguro, no tiene ninguna opción o no aplica", "en desacuerdo" y "completamente en desacuerdo" promediaron un $2 \%$.

b) Percepción, componente cualitativo, a través de una pregunta abierta sobre la valoración general de la intervención y su posible impacto en su desempeño futuro (Tabla 2):

Desde un punto de vista cualitativo, se plantearon preguntas abiertas en las que se solicitaba a los estudiantes que indicaran las fortalezas y oportunidades de mejora del taller. De estos planteamientos se rescataron declaraciones como: "ahora le encuentro sentido a estudiar odontología", "es la primera vez que nos toca hacer práctica en un ser vivo" (haciendo alusión al uso de simuladores tradicionales), "esto finalmente me va a dar más confianza cuando tenga que atender a mis pacientes en cuarto año", "la práctica me ayuda a entender mejor las situaciones clínicas", "sería bueno integrar más de este tipo de talleres a la carrera con diversas patologías a las que nos vamos a enfrentar", "este taller me ayuda a poder vivir un momento para el cual debo prepararme anticipadamente", "nos permitió integrar conocimientos y debatir". 
Tabla 1. Análisis cuantitativo.

\begin{tabular}{|c|c|c|c|c|c|}
\hline Preguntas & $\mathrm{CD} \%$ & $\mathrm{D} \%$ & $\mathrm{SR} \%$ & $\mathrm{~A} \%$ & CA $\%$ \\
\hline $\begin{array}{l}\text { 1. La práctica con paciente estandarizado (actores) } \\
\text { favorece mis habilidades para desarrollar } \\
\text { comunicación con el paciente }\end{array}$ & 2 & & & & 98 \\
\hline $\begin{array}{l}\text { 2. La práctica con paciente estandarizado refuerza mi } \\
\text { confianza en relación con mis habilidades de } \\
\text { comunicación efectiva con pacientes }\end{array}$ & 2 & & & 5 & 93 \\
\hline $\begin{array}{l}\text { 3. La práctica con paciente estandarizado ayuda a prepararme } \\
\text { mejor para mi desempeño con pacientes reales }\end{array}$ & 2 & & & 3 & 95 \\
\hline $\begin{array}{l}\text { 4. Los actores que participaron en las actividades } \\
\text { de simulación parecían pacientes reales }\end{array}$ & & & 2 & 10 & 89 \\
\hline $\begin{array}{l}\text { 5. Recibí retroalimentación útil para mejorar mi } \\
\text { desempeño durante la actividad de simulación }\end{array}$ & & 2 & & 7 & 92 \\
\hline $\begin{array}{l}\text { 6. Me sentí cómodo participando en el taller } \\
\text { de paciente estandarizado }\end{array}$ & & 2 & & 20 & 79 \\
\hline $\begin{array}{l}\text { 7. El trabajo con paciente estandarizado debiera } \\
\text { ser un componente obligatorio en la enseñanza } \\
\text { de las carreras de la salud }\end{array}$ & 2 & & 2 & 10 & 87 \\
\hline 8. Deseo tener más actividades con pacientes estandarizados & 2 & & & 5 & 93 \\
\hline
\end{tabular}

Tabla 2. Análisis cualitativo de frecuencia.

\begin{tabular}{lc} 
Desempeños & $\begin{array}{c}\text { Frecuencia } \\
\text { (No respuestas) }\end{array}$ \\
\hline 1. Autoeficacia & 13 \\
2. Integración del conocimiento & 23 \\
3. Motivación & 23 \\
4. Acercamiento temprano & 27 \\
$\quad$ a la profesión & \\
5. Logro de desempeños & 30 \\
\hline
\end{tabular}

Fuente: Elaboración propia.

El procesamiento de esta información se realizó a través de la codificación para permitir asignar unidades de significado a la información descriptiva obtenida de las preguntas abiertas anexas a la encuesta, para generación y posterior análisis de una tabla de frecuencia organizada con los descriptores autoeficacia, integración del conocimiento, motivación, acercamiento temprano a la profesión y logro de desempeños; estos dos últimos fueron los que ocuparon la mayor cantidad de declaraciones, 30 y 27 , respectivamente.

c) Cumplimiento de los desempeños esperados, a través de una lista de cotejo diseñada para tal fin (Tabla 3):

Por medio de la observación y certificación del evaluador, y siguiendo la pauta de los desempeños esperados en la intervención, se obtuvo que la mayoría de los estudiantes pudieron realizar el diagnóstico de la patología pulpar asociada al caso $(90 \%)$, orientada al componente cognitivo del ejercicio. Con respecto al manejo y sistematicidad de la ficha clínica, relacionado con el componente de aplicación, el $70 \%$ de los estudiantes alcanzaron un indicador positivo, mientras que el desempeño relacional indicado como "empatía con el paciente" alcanzó solamente al $40 \%$ de los estudiantes involucrados. 


\begin{tabular}{lc}
\multicolumn{1}{c}{$\begin{array}{c}\text { Tabla 3. Cumplimiento de } \\
\text { desempeños esperados. }\end{array}$} \\
\hline Componente & Porcentaje \\
\hline 1. Cognitivo & 90 \\
2. Procedimental & 70 \\
3. Actitudinal & 40 \\
\hline
\end{tabular}

Fuente: Elaboración propia.

\section{DISCUSIÓN}

La seguridad del paciente es, desde hace ya varios años, un tema de importancia en la práctica de las profesiones de la salud; esto se ha extrapolado a las etapas de formación de los estudiantes de algunas de estas carreras. ${ }^{8}$ Aprender haciendo ha sido, desde siempre, una experiencia altamente enriquecedora desde el punto de vista educacional; ayuda a cerrar el ciclo del aprendizaje y se encuentra, por ende, en las escalas más altas de la taxonomía de Bloom. La importancia de evolucionar durante las etapas de formación de novato a experto sin poner en riesgo la seguridad de los pacientes ha sido una de las situaciones que ha impulsado la utilización de diferentes niveles de simulación en el desarrollo y práctica de habilidades procedimentales. El nuevo enfoque basado en competencias propone estrategias de formación susceptibles de favorecer la construcción gradual de los conocimientos en el estudiante, tomando en cuenta los elementos afectivos, cognitivos y metacognitivos de los mismos. ${ }^{9,10}$

En Odontología, la gran mayoría de las actividades prácticas preclínicas se desarrolla a partir de simulación de baja fidelidad con simuladores part-task o fantomas, donde es posible practicar diversas acciones clínicas procedimentales, y que por mucho tiempo han cumplido de manera satisfactoria con el desarrollo de las competencias declaradas en el perfil de egreso de los estudiantes de Odontología. ${ }^{11}$ El mundo laboral ha cambiado y esto se ha transmitido al ámbito educativo, se ha modificado el perfil de egreso y se han agregado competencias actitudinales que la simulación de baja fidelidad no permite desarrollar ni medir.

La oportunidad de agregar un paciente estandarizado a la práctica de una acción preclínica permite al estudiante integrar varias habilidades en una misma experiencia. En la Universidad de Arizona se incorporó la simulación con paciente estandarizado para complementar las actividades prácticas de laboratorio en la confección de prótesis totales; los estudiantes encontraron que esto les permitió mejorar la comprensión de la actividad realizada. ${ }^{5}$ Nosotros hallamos una situación similar, donde los estudiantes manifestaron una alta valoración del desarrollo de habilidades de comunicación (98\%) y la preparación para el desempeño con pacientes reales. En el área de las destrezas actitudinales, Wagner y sus colaboradores realizaron una actividad donde se incluyó la experiencia de un paciente estandarizado para la evaluación de habilidades comunicacionales en los estudiantes; se lograron resultados satisfactorios en las capacidades para obtener información médica y dental relevante. ${ }^{7}$ Estos datos son similares a los reportados en este estudio, donde $90 \%$ de los estudiantes lograron recabar signos y síntomas clínicos para lograr el resultado de aprendizaje de realizar el diagnóstico pulpar. Al igual que la experiencia antes mencionada, donde se evaluó la capacidad para realizar pesquisas del componente sicosocial del paciente, se detectó que los estudiantes tenían dificultad para preguntar ciertos tópicos personales a los pacientes, situación que podría replicarse en nuestra experiencia y ser la causa de que sólo 40\% haya logrado el desempeño relacional indicado como "empatía con el paciente". Por ello, este resultado nos permite reforzar que estas competencias se van desarrollando de manera gradual y tras la retroalimentación de lo observado por parte del evaluador; de ahí la importancia de la inserción temprana para disminuir estas brechas. Esta gradualidad en el desarrollo de estas experiencias también fue determinada por Wagner y su equipo, donde un grupo de estudiantes realizó tres experiencias con paciente estandarizado y se observó que la calidad del desempeño aumentó gradualmente. ${ }^{7}$

En el análisis cualitativo, destacó la valoración que tuvieron estos estudiantes sobre la confianza y el desarrollo de competencias 
comunicacionales para poder relacionarse con pacientes reales. La inserción en ciclos preclínicos de escenarios de mayor fidelidad y el entrenamiento sistematizado en aspectos relacionales a través de la simulación clínica pueden impactar de manera positiva en la habilidad comunicacional, lo que refuerza una buena relación odontólogo-paciente. Autores como McKenzie y su grupo evaluaron a una cohorte de estudiantes que realizaron experiencias de simulación clínica con paciente estandarizado en el segundo año de la carrera, a través de la observación de su desempeño clínico con los pacientes dos años después, encontraron que existían diferencias en las etapas de comunicación del plan de tratamiento, donde los estudiantes que tuvieron contacto con pacientes estandarizados eran capaces de ofrecer mayor información u opciones de tratamiento, lo que probablemente estuvo asociado a la capacidad de retener y desarrollar este concepto entregado como parte fundamental de las habilidades comunicacionales durante la entrevista clínica. ${ }^{6,12}$

En cuanto a la percepción cualitativa de los estudiantes, el logro de los desempeños y el acercamiento temprano a la profesión fueron los aspectos mejor valorados. Los estudiantes actuales aprenden mejor a través de experiencias que aseguren el aprendizaje significativo, lo que les permite experimentar lo aprendido en el campo laboral, autoevaluarse e identificar su nivel de conocimiento, mejorar sus habilidades técnicas, aumentar la seguridad, confianza, razonamiento crítico y toma de decisiones. ${ }^{9,13}$

\section{CONCLUSIONES}

En el análisis cuantitativo de la experiencia con paciente estandarizado, el desarrollo de habilidades de comunicación (98\%) obtuvo la valoración más alta por parte de los estudiantes, seguido de la preparación para el desempeño con pacientes reales $(95 \%)$, la confianza en sus capacidades comunicativas (93\%) y el deseo de contar con más actividades de este tipo (93\%). Dentro de los ítems menos valorados se encontró la comodidad con la estrategia (79\%).

Dentro del análisis cualitativo a través de una pregunta abierta, se rescataron afirmaciones como: "es la primera vez que nos toca hacer práctica en un ser vivo" y "la práctica me ayuda a entender mejor las situaciones clínicas".

En relación con el cumplimiento de los desempeños esperados, a través de una lista de cotejo, el diagnóstico de la patología pulpar asociada al caso fue logrado por $90 \%$ de los estudiantes, mientras que el desempeño relacional indicado como "empatía con el paciente" alcanzó solamente al $40 \%$ de los estudiantes.

\section{REFERENCIAS}

1. Jaramillo G, Caro H, Parra ZA, Bedoya JP, Pabón EA, Mejía MC. Dispositivos desencadenantes de estrés y ansiedad en estudiantes de Odontología de la Universidad de Antioquia. Revista Facultad de Odontología Universidad de Antioquia. 2009; 20: 49-57.

2. Armijo S, Jabes N, Larsen F. Evaluación del impacto de una ayudantía de Simulación Clínica en el logro de competencias clínicas intermedias en estudiantes de tercer año de medicina. Revista de Educación en Ciencias de la Salud, 2014; 10.

3. Okuda Y, Bryson EO, DeMaria S Jr, Jacobson L, Quinones J, Shen B et al. The utility of simulation in medical education: what is the evidence? Mt Sinai J Med. 2009; 76: 330-343.

4. Ortega Al, Casanova II, Pertuz RA, Cárdenas EM. Tendencias tecnológicas: simulación en la formación odontológica. Rev Ciencia Odont. 2010; 7: 116-128.

5. Johnson GM, Halket CA, Ferguson GP, Perry J. Using standardized patients to teach complete denture procedures in second year of dental school. J Dent Educ. 2017; 81: 340346.

6. McKenzie CT, Tilashalski KR, Peterson DT, White ML. Effectiveness of standardized patient simulations in teaching clinical communication skills to dental students. J Dental Educ. 2017; 81: 1179-1186.

7. Wagner J, Arteaga S, D'Ambrosio, J, Hodge CE, loannidou E, Pfeiffer CA et al. A patient-instructor program to promote dental students' communication skills with diverse patients. J Dent Educ. 2007; 71: 1554-1560.

8. Obadan EM, Ramoni RB, Kalenderian E. Lessons learned from dental patient safety case reports. J Am Dent Assoc. 2015; 146: 318-326.

9. March AF. La evaluación orientada al aprendizaje en un modelo de formación por competencias en la educación universitaria. REDU: Revista de Docencia Universitaria. 2010; 8: 11-34.

10. Amaya AA. Simulación clínica: aproximación pedagógica de la simulación clínica. Universitas Médica, 2010; 51: 204-211.

11. Perry S, Bridges SM, Burrow MF. A review of the use of simulation in dental education. Simul Healthc. 2015; 10: 31-37.

12. Logan HL, Muller PJ, Edwards Y. Using standardized patients to assess presentation of a dental treatment plan. J Dental Educ. 1999; 63: 729-737.

13. Castillo-Arcos LC, Maas-Góngora L. Percepción de satisfacción de los estudiantes de Enfermería en el uso de la simulación clínica. Ra Ximhai. 2017; 13: 63-76.

\author{
Correspondencia: \\ Mario Zúñiga-Mogollones \\ Facultad de Odontología \\ Universidad San Sebastián \\ Bellavista 7, Santiago, Chile. \\ 8420524 \\ E-mail: mario.zuniga@uss.cl
}

